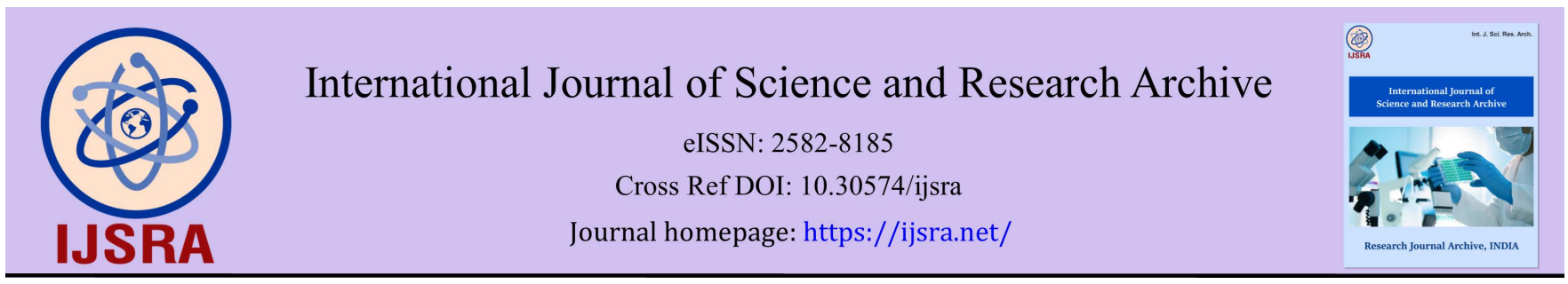

(CASE REPORT)

Check for updates

\title{
Persistent otitis media with effusion: An unusual type of nasopharyngeal adenocarcinoma
}

Athina Zarachi 1, *, Zoi Evangelou 2, Angelos Liontos 3, Stefania Gkoura 4, Alkistis Tsikou 1, Ioannis Kastanioudakis ${ }^{1}$ and Ioannis Komnos ${ }^{1}$

${ }^{1}$ Department of Otorhinolaryngology, Head and Neck Surgery, Faculty of Medicine, School of Health Sciences, University of Ioannina, Ioannina, Greece.

${ }^{2}$ Department of Pathology, Faculty of Medicine, School of Health Sciences, University of Ioannina, Ioannina, Greece.

${ }^{3}$ Faculty of Medicine, Department of Internal Medicine, School of Health Sciences, University of Ioannina, Ioannina, Greece.

${ }^{4}$ Department of Oncology, Faculty of Medicine, School of Health Sciences, University of Ioannina, Ioannina, Greece.

International Journal of Science and Research Archive, 2021, 03[02], 113-117

Publication history: Received on 22 August 2021; revised on 26 September 2021; accepted on 28 September 2021

Article DOI: https://doi.org/10.30574/ijsra.2021.3.2.0145

\begin{abstract}
We present the case of a 41-year-old male patient that examined in the outpatient ENT Department of our hospital, reported a persistent otitis media for about six months. Despite medical therapy, his symptom did not improve. The suspicious of a nasopharyngeal mass was raised and the rhino endoscopy revealed a mucosal projection in the left fossa of the Rosenmuller. Computed tomography (CT) of temporal bone pneumatization showed a left mastoid cell occupation and a small projection in the left fossa of the Rosenmuller. The histological examination result of a biopsy of the lession that prepared under local anesthesia, revealed nasopharyngeal adenoid cystic carcinoma. Magnetic resonance imaging indicated the extent of the tumor. The positron emission tomography/computed tomography (PET/CT) scan staged the patient and concluded the diagnosis. The patient underwent both surgery and radiotherapy and addressed oncologists and radiotherapists for further treatment. The patient was diagnosed with lung and kidney metastasis. Adenoid cystic carcinoma is a rare type of adenocarcinoma with ominous prognosis. We always have to raise the suspicious of nasopharyngeal carcinoma, in patients with persistent otological symptoms, that do not improve despite therapy.
\end{abstract}

Keywords: Adenocarcinoma; Adenic cystic carcinoma; Nasopharynx; otitis media; Effusion; Otalgia; Persistent symptoms

\section{Introduction}

Nasopharyngeal carcinoma (NPC) occurs in the epithelial lining of the nasopharynx, and has a variety of subtypes, pathologically. The majority of NPC cases involve squamous cell carcinoma [NPSC], while additional pathological types of NPC include adenocarcinoma, lymphoma and sarcomas, constituting less than 5\% of all NPC cases[1, 2]. Specifically, nasopharyngeal adenocarcinoma (NPAC) is histologically characterised by the presence of a glandular structure and production of mucus, and reported to occupy $0.48 \%$ in all types of NPC. According to classification by the World Health Organization, NPAC can be divided into common type and salivary gland type cases. Subtypes of nasopharyngeal salivary gland-type adenocarcinoma include the mucoepidermoid adenocarcinoma, the adenoid cystic adenocarcinoma , the polymorphous low-grade adenocarcinoma and the malignant mixed tumor, that come from different cell population and appear different prognosis[3-6]. Nasopharyngeal adenoid cystic carcinoma (NACC) is a rare tumor that represents approximately $1 \%$ of all carcinomas of the head and neck. NACC tends to be locally aggressive, demonstrate perineural invasion and appears ominous prognosis[7-10]. Except the type of nasopharyngeal carcinoma, overall

${ }^{*}$ Corresponding author: Athina Zarachi

Department of Otorhinolaryngology, Head and Neck Surgery, Faculty of Medicine, School of Health Sciences, University of Ioannina, Ioannina, Greece.

Copyright $@ 2021$ Author[s] retain the copyright of this article. This article is published under the terms of the Creative Commons Attribution Liscense 4.0. 
patients with a nasopharyngeal neoplasm frequently present with at least four symptoms including nasal symptoms (epistaxis, nasal obstruction), neck masses, otological symptoms [tinnitus, deafness] and cranial nerve palsies. Despite the fact that nasopharyngeal tumors usually infiltrate the eustachian tube muscles and do not involve the eustachian tube opening, the association of nasopharyngeal carcinoma with middle ear effusion relates with poor middle ear ventilation[11]. We present the case of a patient reported otological symptoms for about six months. His symptoms did not improve despite therapy, so he decided to address our clinic, for further investigation.

\section{Case report}

A 41-year-old male patient examined in the outpatient ENT Department of our hospital, during January of 2019. He reported left ear pain, hearing loss and ear fullness for about six months. He had already addressed outpatient doctors twice. Both doctors set the diagnosis of otitis media with effusion, because of Eustachian tube dysfunction and proposed antibiotics and decongestant nasal spray as therapy. The patient followed the recommended therapy, but despite the implicit improvement of symptoms, he did not improve. According to the patient's medical history, he did not receive any medication. Through clinical examination, otoscopically, the left ear drum showed up otitis media with effusion. No lymph nodes were found in the left neck and post auricular area. The rhinoednoscopy of the nasopharynx revealed a small projection in the left fossa of Rosenmuller (Figure 1).

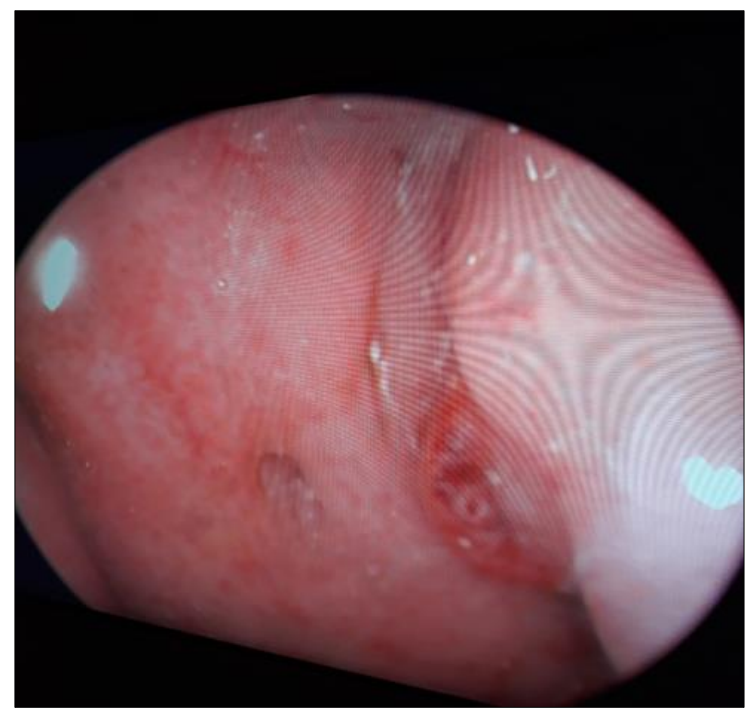

Figure 1 Mucosal projection in the left fossa of Rosenmuller

For the evaluation of hearing loss, the $512-\mathrm{Hz}$ tuning fork was used. The Rinne test was normal, while in the Weber test, sound lateralized to the left ear, suggesting conductive loss in the left ear. The audiogram confirmed the conductive hearing loss in the left ear. The tympanogram [type B] revealed middle ear effusion. Computed tomography (CT) of temporal bone pneumatization showed a left mastoid cell occupation and a small mucosal projection in the left fossa of the Rosenmuller (Figure 2).

A biopsy of the lesion performed, under local anesthesia. The insertion of ventilation tube in the left ear was held, in order to cure the middle ear effusion. Biopsy of the lesion was fixed in formalin and sent to a referral laboratory for histological preparation and examination. An immunehisto-chemical analysis with molecular detection of human papillomavirus [HPV] ended up that the carcinoma morphologically looks like adenoid cystic carcinoma of the nasopharynx [Fingure 3].A magnetic resonance imaging (MRI) showed up the extent of tumor ( Figure 4,5). Through a computed tomography chest, nodular lesions were located in the lobe of the left lung. The findings through a positron emission tomography/computed tomography (PET/CT) scan were compatible with the previous findings through CT and MRI . The patient combined modality therapy consisting of surgery and radiotherapy. A lung biopsy revealed metastatic lung cancer, so the patient underwent a partial left lung lobectomy. Six months later, the patient was diagnosed with a left kidney metastasis, so he underwent nephrectomy. A repeated follow up examination is proposed for the patient since now. 


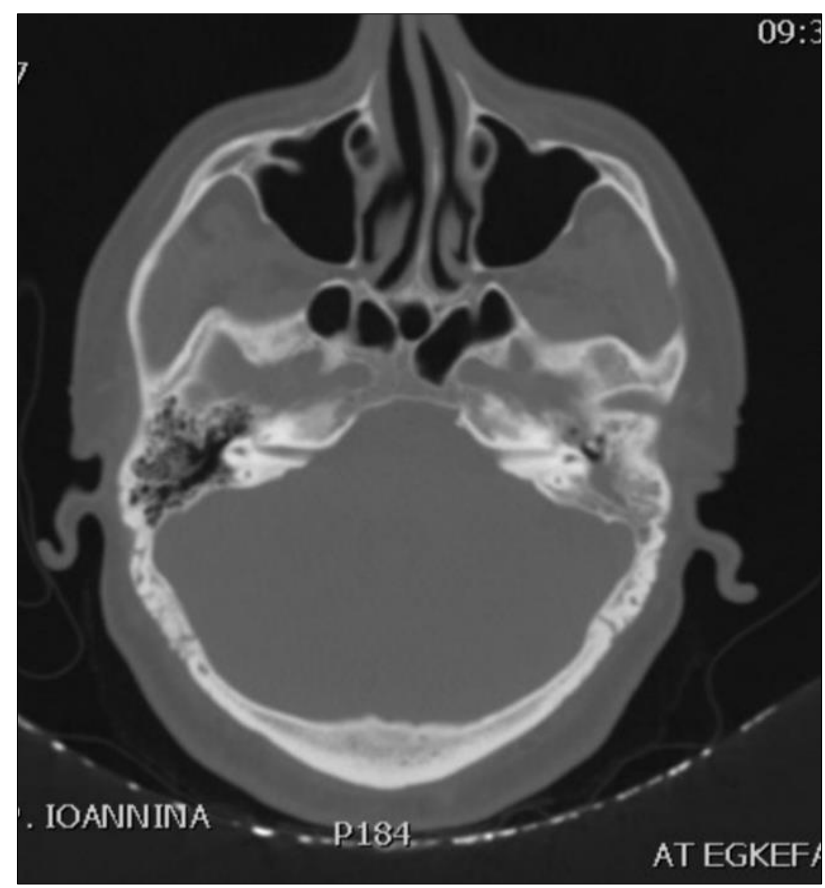

Figure 2 Computed tomography (CT) of temporal bone pneumatization shows a left mastoid cell occupation and a small mucosal projection in the area of the Rosenmüller fossa

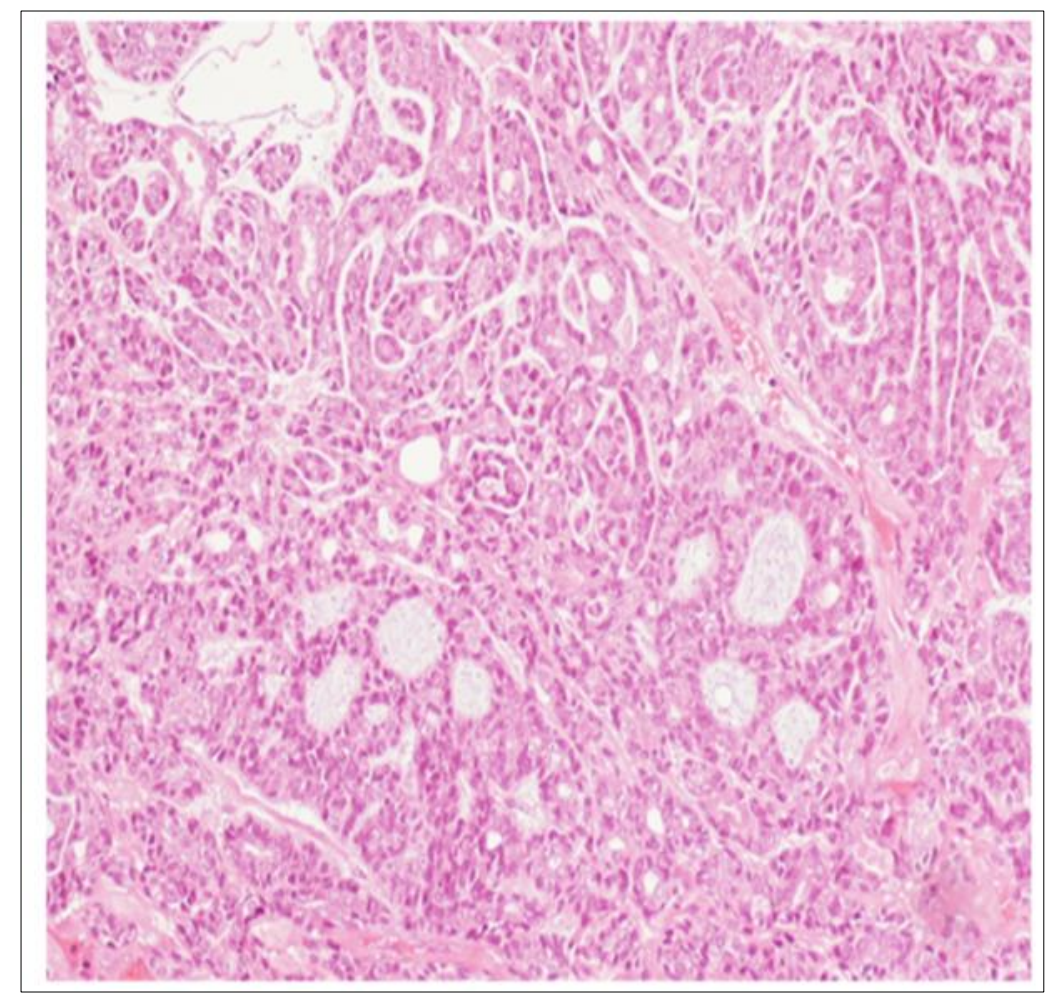

Figure 3 This cellular neoplasm has a tubular and cribriform architecture. The structures are lined by two types of cells with moderate atypia, one with dark angulated nuclei and scanty cytoplasm which stain positive to p63 protein and one larger population with a light-colored round to oval nuclei and visible nucleoli which stain positive for c-kit and CK7. The sharply punched-out spaces are filled with basophilic matrix. 


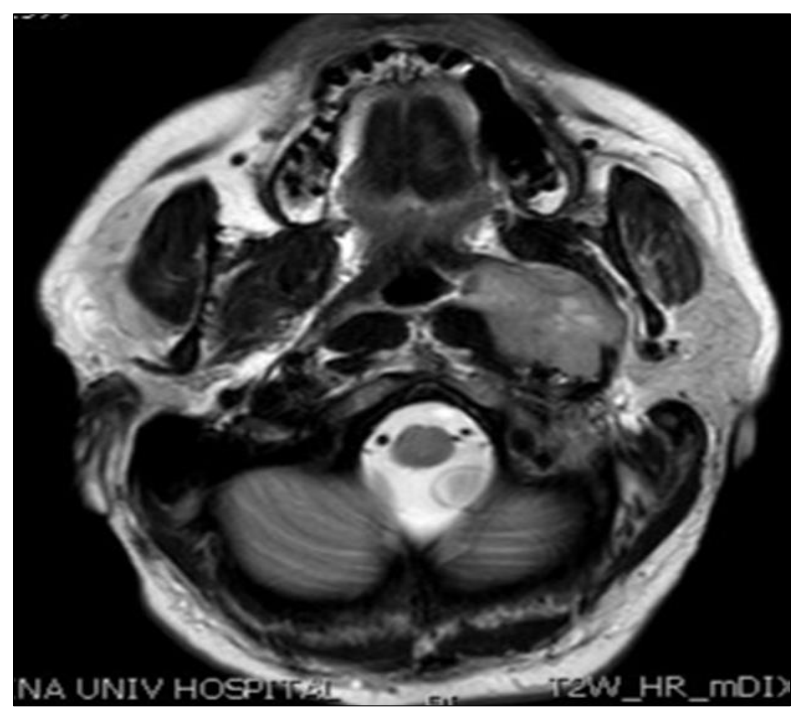

Figure 4 Magnetic resonance imaging shows the extent of nasopharyngeal carcinoma

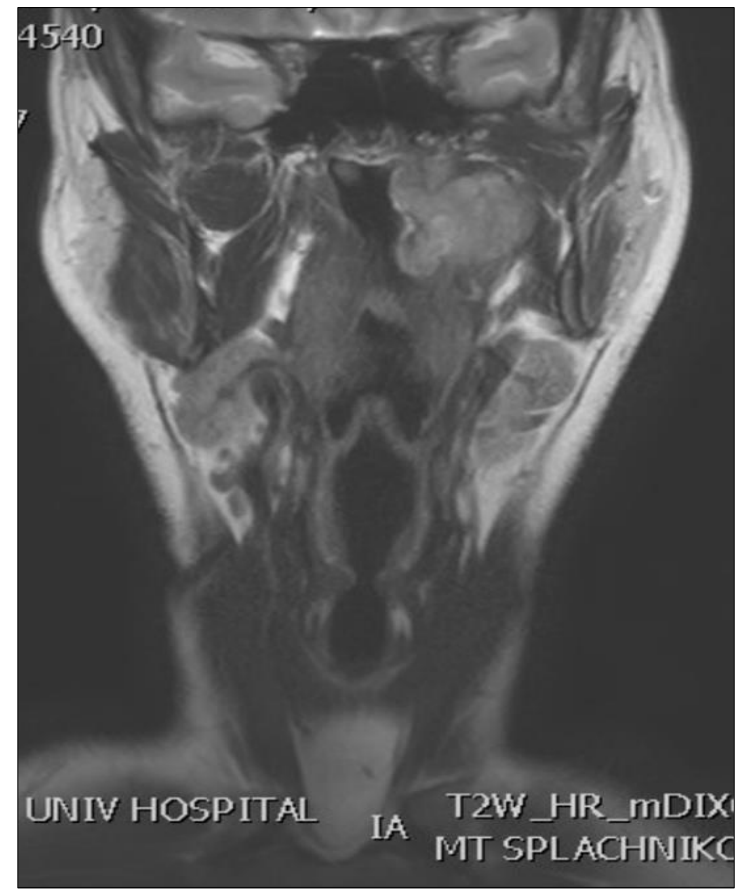

Figure 5 Magnetic resonance imaging shows the extent of nasopharyngeal carcinoma

\section{Discussion}

Nasopharyngeal adenocarcinoma is a rare tumor of the nasopharynx. It appears local invasion in the roof or posterior wall of nasopharynx, in the lateral nasopharyngeal wall, in parapharyngeal space, or it can be extended up to the skull base and the cranial nerves. Skull base and intracranial spread may occur through the foramen lacerum, while posterolateral spread may occur through the sinus of Morgagni. The adenoid cystic carcinoma is one of the subtypes of nasopharyngeal salivary gland-type adenocarcinoma with common presenting symptoms: epistaxis, nasal obstruction, tinnitus, hearing loss. Other common presenting symptoms are cervical lymphadenopathy, otalgia, the sensation of an obstructed ear, or conductive hearing loss due to serous otitis, caused by eustachian tube obstruction.[1,10]. Compared to the common type of adenocarcinoma, NACC is much most aggressive and appears bad prognosis. Radiotherapy alone (RT), surgery alone, combined RT - surgery or chemotherapy, are all included in treatment approaches of NACC. The 
appropriate treatment will be determined by the extent and stage of the disease [12]. The location, shape, margin, signal intensity, lesion texture, contrast enhancement patterns, local invasion, and cervical lymphadenopathy of such a tumor can be evaluated through a magnetic resonance tomography[13].Clinical factors of interest, are the perineural invasion and cranial nerve invasion and must be considered for the treatment appropriate for each clinical case [9, 14].

\section{Conclusion}

Many factors can cause eustachian tube obstruction and prolonged serous otitis. Nasopharyngeal carcinoma is a rare but possible cause. In every case of persistent otitis media with effusion, further clinical investigation through rhinoendoscopy and imaging of the nasopharynx must be performed.

\section{Compliance with ethical standards}

\section{Disclosure of conflict of interest}

The authors declare no conflicts of interest regarding the publication of this paper.

\section{Statement of informed consent}

Informed consent was obtained from all individual participants included in the study.

\section{References}

[1] Dubrulle F, Souillard R, Hermans R. Extension patterns of nasopharyngeal carcinoma. European Radiology. 2007; 17[10]: 2622-2630.

[2] Zong YS, Zheng WJ. A morphologic and follow-up study on the nasopharyngeal lymphoid hyperplasia and its relation to the cancer. Chin Med J [Engl]. 1989; 102: 625-9.

[3] Zong YS, Zhang RF, Chen ZH. Well differentiated nasopharyngeal adenocarcinoma. Pathologic analysis of 34 cases. Chin Med J [Engl]. 1983; 96: 63-8.

[4] XU T, LI ZM, GU MF, WEI W, ZHANG G, WU QL, HU WH. Primary nasopharyngeal adenocarcinoma: A review. AsiaPacific Journal of Clinical Oncology. 2012; 8[2]: 123-131.

[5] He JH, Zong YS, Luo RZ, et al. Clinicopathological characteristics of primary nasopharyngeal adenocarcinoma. Ai Zheng. 2003; 22: 753-757.

[6] Guo ZM,, Liu WW, He JH. A retrospective cohort study of nasopharyngeal adenocarcinoma: a rare histological type of nasopharyngeal cancer. Clinical Otolaryngology. 2009; 34[4]: 322-327.

[7] Barrett AW, Speight PM. Perineural invasion in adenoid cystic carcinoma of the salivary glands: A valid prognostic indicator? Oral Oncology. 2009; 45: 936-40.

[8] Tatari MM, Anajar S, Hassnaoui J, Lahjaouj M, Salama K, Rouadi S, MahtarM. Nasopharyngeal adenoid cystic carcinoma, suggestion of therapeutic innovations: A case report and review of literature. Annals of Medicine and Surgery. 2018; 30: 32-35.

[9] Ryckman JM, Kusi Appiah A, Simone CB, Verma V. Treatment approaches for nasopharyngeal adenoid cystic carcinoma. Acta Oncologica. 2018; 57[7]: 995-1001.

[10] Liu TR, Yang AK, Guo X, Li QL, Song M, He JH, Chen FJ. Adenoid Cystic Carcinoma of the Nasopharynx: 27-Year Experience. The Laryngoscope. 2008; 118[11]: 1981-1988.

[11] Sadé J. The nasopharynx, eustachian tube and otitis media. The Journal of Laryngology \& Otology. 1994; 108[02].

[12] Cao CN, Luo JW, Xu GZ, Gao L, Xu ZG, Tang PZ. Management of Nasopharyngeal Adenoid Cystic Carcinoma. Journal of Oral and Maxillofacial Surgery. 2013; 71[4]: e203-e209.

[13] Xue-Wen Liu C-MX, Hui Li,et al. Nasopharyngeal adenoid cystic carcinoma: magnetic resonance imaging features in ten cases. Chin J Cancer. Jan 2012; 31[1]: 19-28.

[14] Xia P, Fu KK, Wong GW, Akazawa C, Verhey LJ. Comparison of treatment plans involving intensity-modulated radiotherapy for nasopharyngeal carcinoma. International Journal of Radiation Oncology*Biology*Physics. 2000; 48[2]: 329-337. 